\title{
An Application of $\beta$-glycosidase to Transformation of Ginsenosides for the Effective Production of Specific Ginsenosides with Biological Efficacy
}

\author{
Youl Her, Young-Chul Lee, Jin-Hwan Oh, Yoon-E Choi, Chang-Woo Lee, Jin-Suk Kim, Hwan Mook Kim, and \\ Ji-Won Yang
}

Received: 12 December 2011 / Revised: 7 February 2012 / Accepted: 7 February 2012

(C) The Korean Society for Biotechnology and Bioengineering and Springer 2013

In the 2012 issue of Biotechnology and Bioprocess Engineering (BBE), an error occurred in the research article: Youl Her, Young-Chul Lee, Jin-Hwan Oh, Yoon-E Choi, Chang-Woo Lee, Jin-Suk Kim, Hwan Mook Kim, and JiWon Yang (2012) An Application of $\beta$-glycosidase to Transformation of Ginsenosides for the Effective Production of Specific Ginsenosides with Biological Efficacy. Biotechnol. Bioprocess Eng. 17: 538-546.

\section{- In the authors's affiliation}

\author{
Original wording: \\ Young-Chul Lee \\ Department of Chemical and Biomolecular Engineering \\ (BK21 program), KAIST, Daejeon 305-701, Korea
}

This should be replaced by:

Youl Her, Young-Chul Lee

Department of Chemical and Biomolecular Engineering (BK21 program), KAIST, Daejeon 305-701, Korea

Received: 25 April 2013

\footnotetext{
Youl Her, Jin-Hwan Oh, Jin-Suk Kim

BTGin Co. Ltd., Daejeon 305-500, Korea

Youl Her, Young-Chul Lee

Department of Chemical and Biomolecular Engineering (BK21 program), KAIST, Daejeon 305-701, Korea

Yoon-E Choi

LED Agri-bio Fusion Technology Research Center, Chonbuk National University, Jeonju 570-752, Korea

Ji-Won Yang*

Advanced Biomass R\&D Center, KAIST, Daejeon 305-701, Korea

Tel: +82-42-350-3924; Fax: +82-42-350-3910

E-mail: jiwonyang@kaist.ac.kr

Chang-Woo Lee

Bioevaluation Center, Korea Research Institute of Bioscience and

Biotechnology, Daejeon 363-883, Korea

Hwan Mook Kim*

College of Pharmacy, Gachon University, Incheon 406-799, Korea

Tel: +82-32-820-4825; Fax: +82-32-820-4829

E-mail: hwanmook@gachon.ac.kr
} 